\title{
Ultrathin Orthorhombic PbS nanosheets
}

Quinten A. Akkerman, ${ }^{\dagger, \ominus}$ Beatriz Martín García, ${ }^{\ddagger}, \ominus$ Joka Buha, ${ }^{\dagger}$ Guilherme Almeida, ${ }^{\dagger}$ Stefano Toso, ${ }^{\dagger \perp}$ Sergio Marras, $"$ Francesco Bonaccorso, ${ }^{\ddagger}$, a Urko Petralanda ${ }^{\dagger}$, Ivan Infante ${ }^{*},+, \mathrm{b}$ and Liberato Manna*†

${ }^{\dagger}$ Nanochemistry Department, ${ }^{\ddagger}$ Graphene Labs, "Materials Characterization Facility, Istituto Italiano di Tecnologia, Via Morego 30, 16163 Genova, Italy

${ }^{\perp}$ Dipartimento di Matematica e Fisica and Interdisciplinary Laboratories for Advanced Materials Physics, Università Cattolica del Sacro Cuore, via Musei 41, I-25121 Brescia, Italy

aBeDimensional Srl., via Albisola 121, 16163 Genova, Italy

bDepartment of Theoretical Chemistry, Vrije Universiteit Amsterdam, De Boelelaan 1083, 1081 HV Amsterdam, The Netherlands

Additional electron microscopy characterization of the PbS nanosheets. As shown in the low magnification TEM images of Figure $\mathrm{S}$, our synthesis protocol using octadecene (ODE) as a solvent leads to the formation of monodisperse nanosheets (NSs) with lateral dimensions of about 180 by $35 \mathrm{~nm}$. Stacks of PbS NSs are also observed, see Figures $\mathrm{S}_{2}$ and $\mathrm{S}_{3}$, with an average thickness of $1.2 \pm 0.3 \mathrm{~nm}$ and NS to NS interdistance of $3.6 \pm 0.3 \mathrm{~nm}$, as displayed in the histograms. To complement the grey scale histogram shown in Figure 1e of the main text, another region (ca. $4.7 \mu \mathrm{m}^{2}$ ) in the TEM grid is analyzed in Figure $\mathrm{S}_{4}$, indicating a series of well-defined intensities, correlating with up to quadrupole stack of PbS NSs.

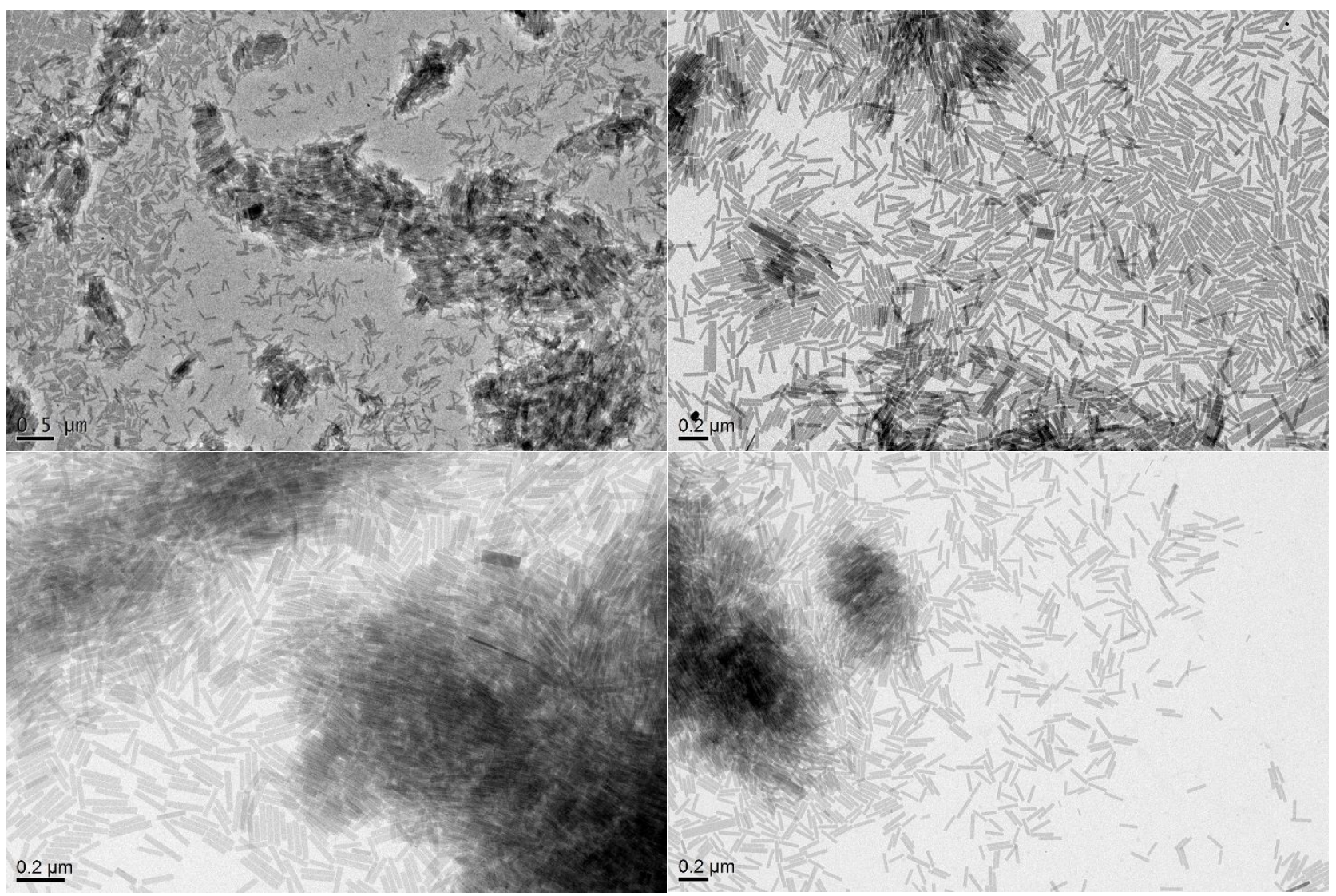

Figure S1. Several large area TEM images of PbS nanosheets (NSs), indicating monodispersity. 


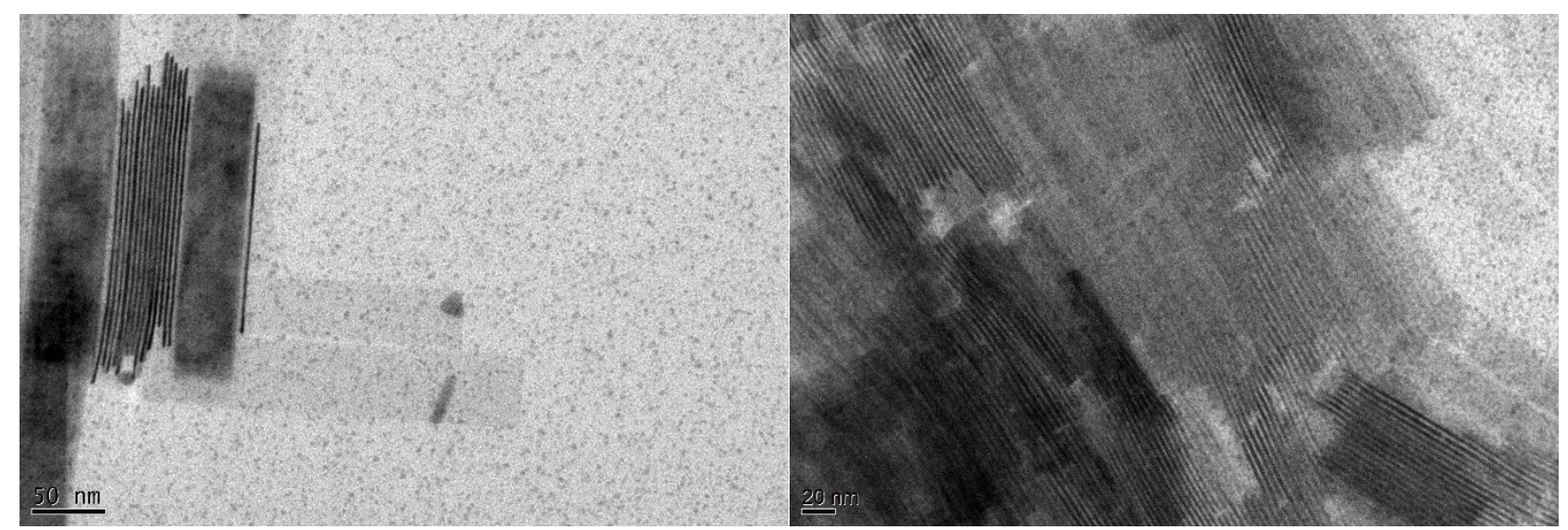

Figure S2. Stacks of PbS NSs oriented side-on towards viewing direction.
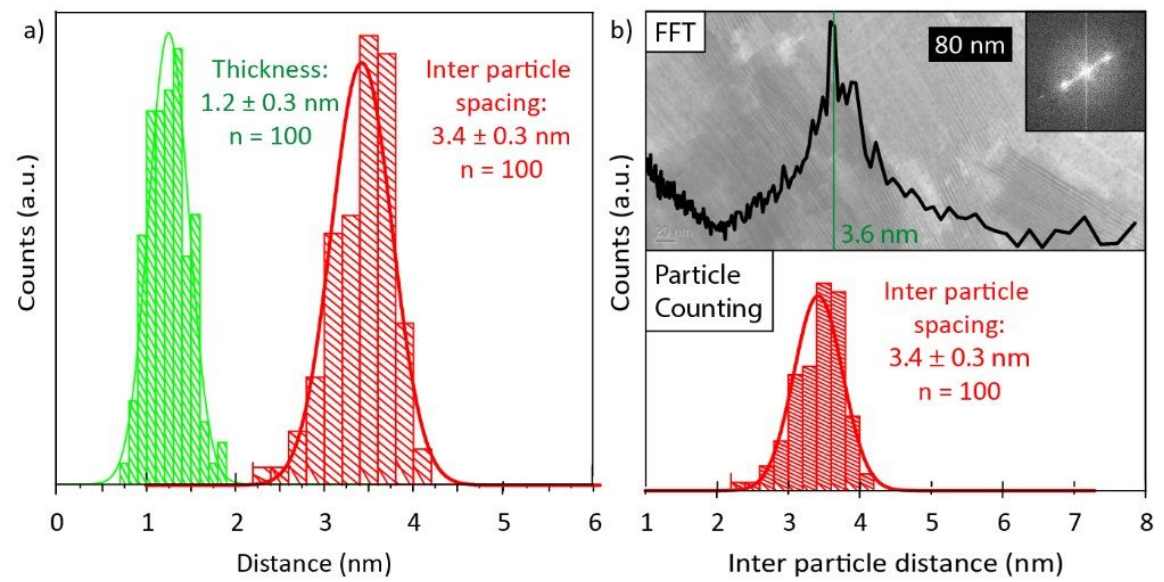

Figure S3. a) PbS NSs thickness and inter particle spacing determined though particle counting. b) Comparison on inter particle distance determined through counting and FFT. Due to the high amount of noise in the FFT, the inter particle distance was determined through particle counting.
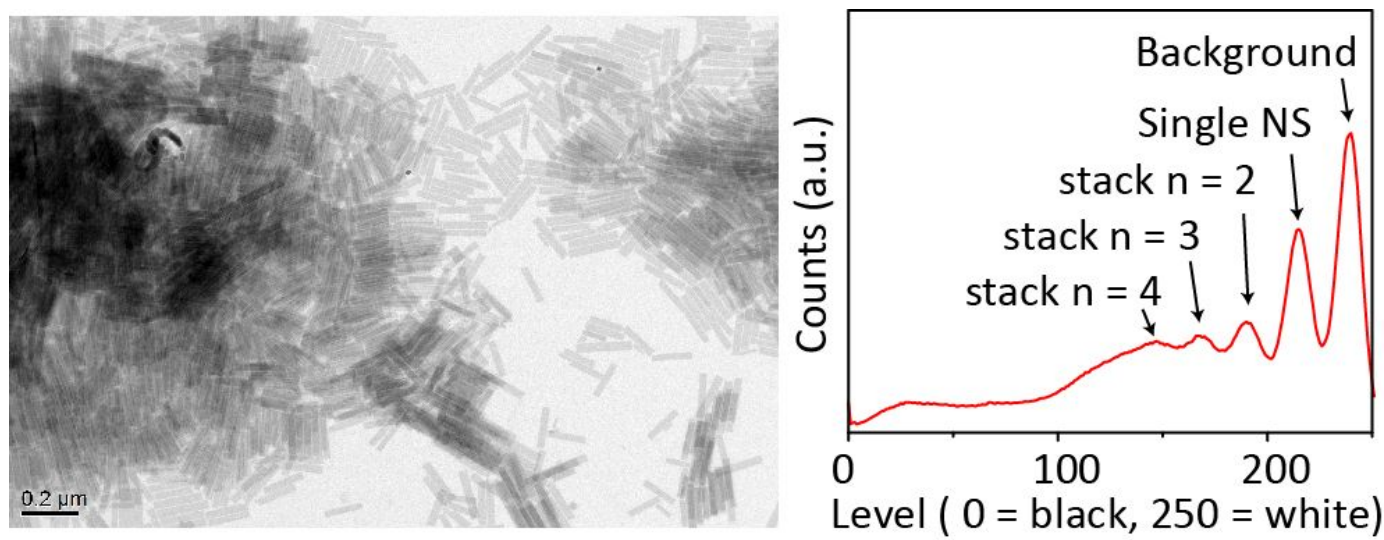

Figure $\mathbf{S}$. Additional histogram of PbS NSs. 
Influence of the reaction parameters: PbS NS size variation. By modifying the reaction temperature, amount of reagents and quenching speed, it is possible to control the lateral dimensions of the PbS NSs, as can be seen in Table Si and the corresponding TEM images of the PbS NSs for the different conditions tested, see Figures $\mathrm{S}_{5}$-S9.

Table S1. Reaction conditions used to obtain different sizes of PbS NSs.

\begin{tabular}{|c|c|c|c|c|c|c|}
\hline Size $(\mathbf{n m})$ & $\begin{array}{c}\text { Pb(SCN) } \\
(\mathbf{m m o l})\end{array}$ & $\begin{array}{c}\text { Reaction temperature } \\
(\mathbf{0} \mathbf{C})\end{array}$ & ODE $(\mathbf{m L})$ & $\begin{array}{c}\text { OLAM } \\
(\mathbf{m L})\end{array}$ & OA (mL) & quenching \\
\hline $25^{*}$ & 0.1 & 150 & 10 & 0.125 & 0.250 & Ice bath \\
\hline $70^{* *}$ & 0.2 & 165 & 10 & 0.125 & 0.250 & Water bath \\
\hline 140 & 0.1 & 165 & 10 & 0.125 & 0.125 & Water bath \\
\hline $180^{* * *}$ & 0.1 & 165 & 10 & 0.125 & 0.250 & Water bath \\
\hline 400 & 0.1 & 165 & 10 & 0.125 & 0.50 & $\begin{array}{c}\text { By removing heat } \\
\text { source only }\end{array}$ \\
\hline $1000^{* * * *}$ & 0.2 & 180 & 5 & 0.25 & 0.5 & $\begin{array}{c}\text { By removing heat } \\
\text { source only }\end{array}$ \\
\hline
\end{tabular}

${ }^{*} 25 \mathrm{~nm}$ PbS NSs are very low in yield and difficult to separate from growth medium.

** $70 \mathrm{~nm} \mathrm{PbS}$ NSs obtained at the limit of solubility of $\mathrm{Pb}(\mathrm{SCN})_{2}$ precursor in the reaction medium.

*** $180 \mathrm{~nm}$ nanosheets were used for structural, optical and conductivity characterization

****1000 nm PbS NS samples contain thicker (PbS) NCs.

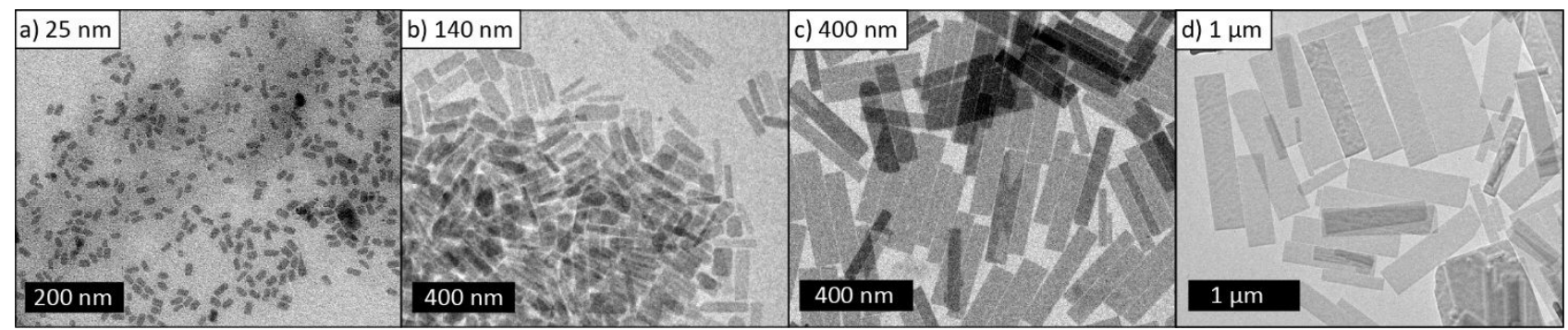

Figure $\mathrm{S}_{5}$. PbS NSs of different lateral sizes ranging from f) 25 to g) 140 to h) $400 \mathrm{~nm}$ up to i) $1 \mu \mathrm{m}$ by controlling the reaction temperature and growth time.
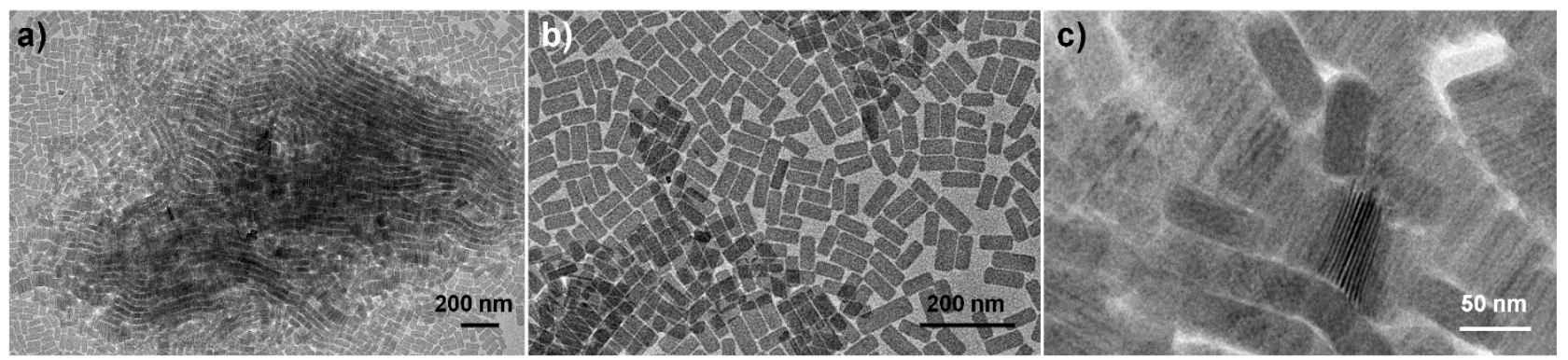

Figure S6. TEM images of the $70 \times 30 \mathrm{~nm} \mathrm{PbS}$ nanosheets obtained increasing the amount of $\mathrm{Pb}(\mathrm{SCN})_{2}$ precursor in the reaction. The last TEM image shows a stack of PbS NSs oriented side-on towards viewing direction, from which it is possible to estimate a $1.2 \mathrm{~nm}$ NS thickness. 


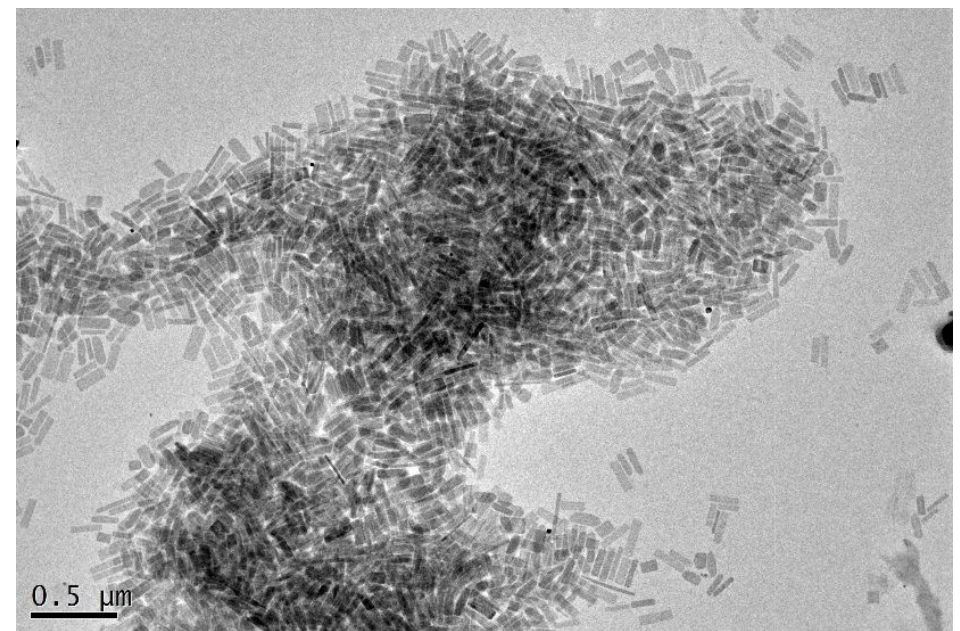

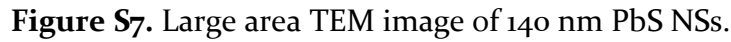

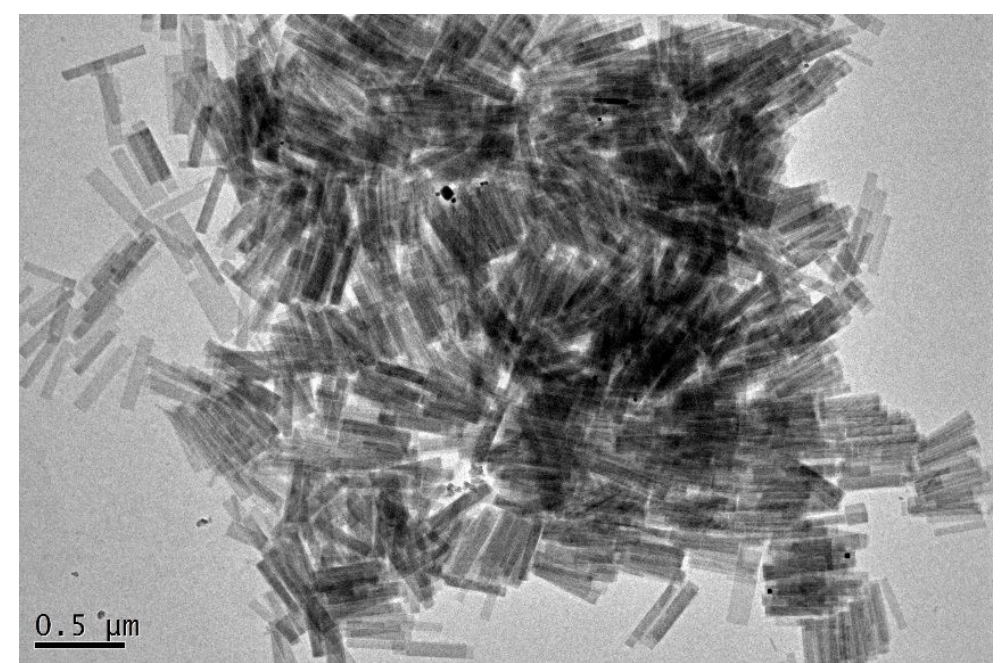

Figure S8. Large area TEM image of $400 \mathrm{~nm}$ NSs.
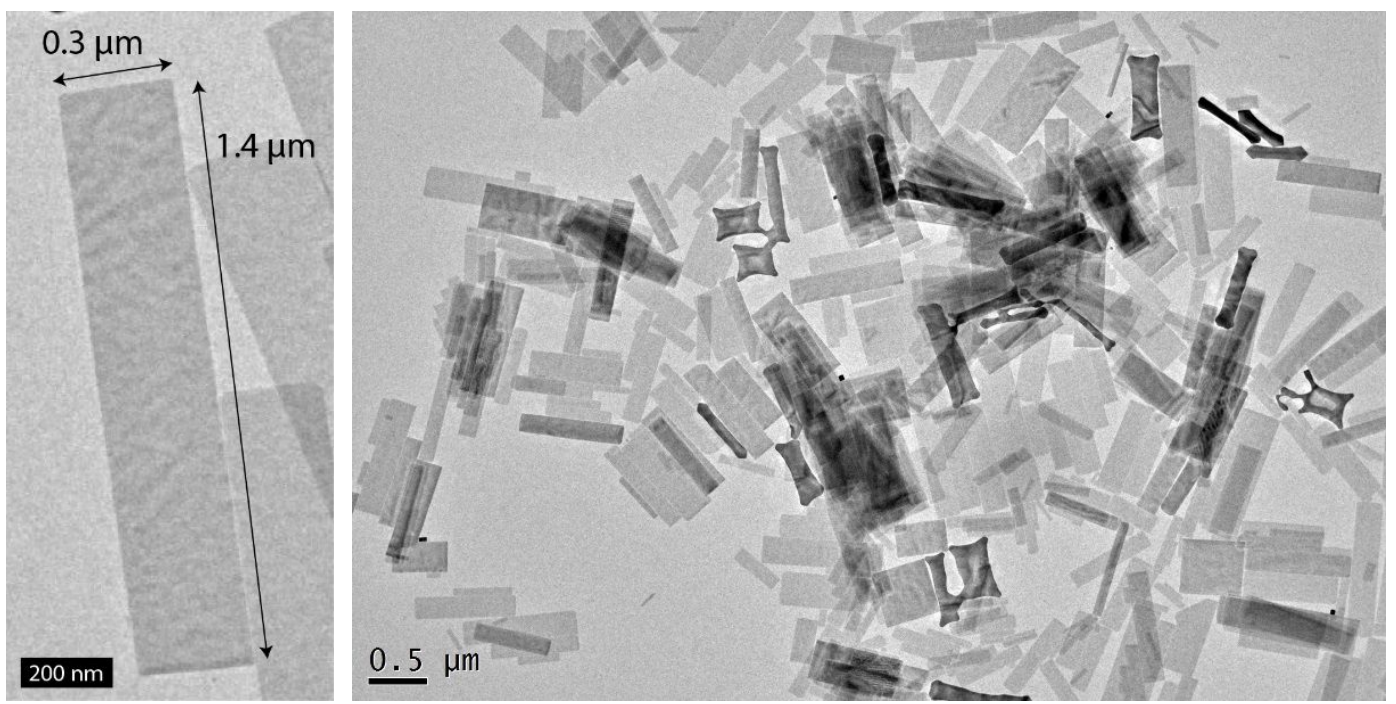

Figure S9. TEM image of PbS NSs up to $1 \mu \mathrm{m}$ in length, showing also the formation of thicker PbS nanoparticles. 
Complementary X-Ray diffraction characterization data. For clearer comparison than in Figure $2 \mathrm{a}$ of the main text, the $\mathrm{X}$-Ray diffraction (XRD) pattern of drop cast PbS NSs and the rock-salt reference pattern of PbS are displayed in Figure Sio; the mismatch with some of the characteristic rock-salt peaks is clearly visible. Additional calculated XRD patterns obtained by changing the NS size along the $a, b$ and $c$ axes directions are included in Figure Sir. Those indicate that the NSs have a preferential growth along the [oo1] direction. Low angle XRD of PbS NSs in Figure S12 allowed us to determine an average inter-NSs stacking thickness of $5.4 \mathrm{~nm}$.

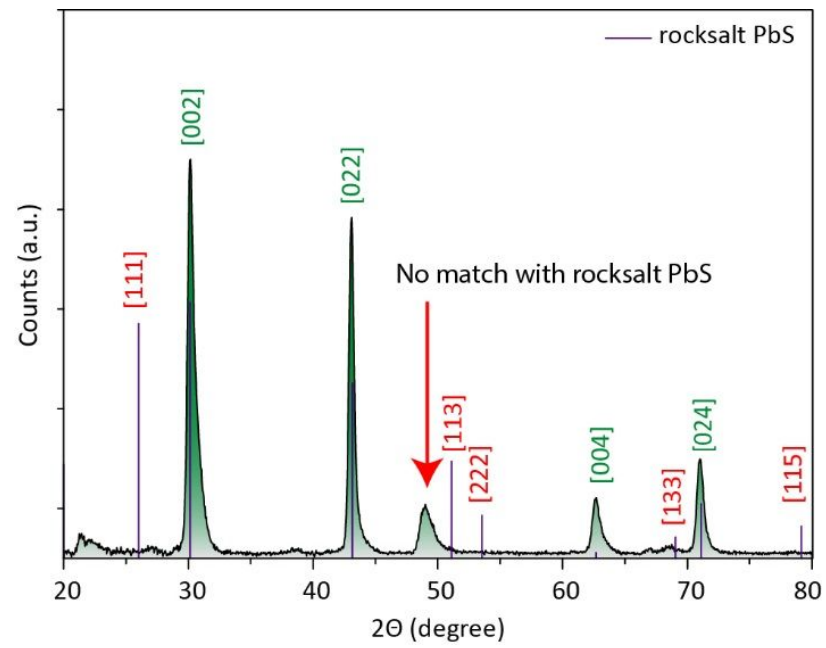

Figure S1o. In plane XRD pattern of drop cast PbS NSs compared to rock-salt XRD pattern of PbS.

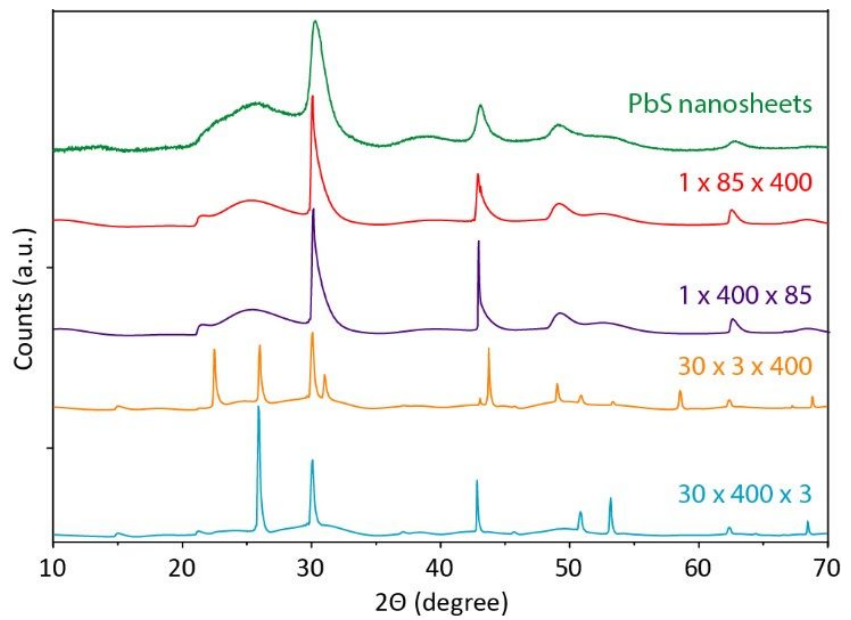

Figure S11. Additional calculated nano powered XRD patterns with orthorhombic PbS unit cell with alternating a $\mathrm{x} b \mathrm{x} \mathrm{c}$ dimensions including: 1 × $85 \times 400(12 \times 36 \times 168 \mathrm{~nm})$, $1 \times 400 \times 85(12 \times 169 \times 36 \mathrm{~nm})$, $30 \times 3 \times 400(36 \times 13 \times 168 \mathrm{~nm}), 30 \times 400$ x $3(36 \times 169 \times 13 \mathrm{~nm})$.

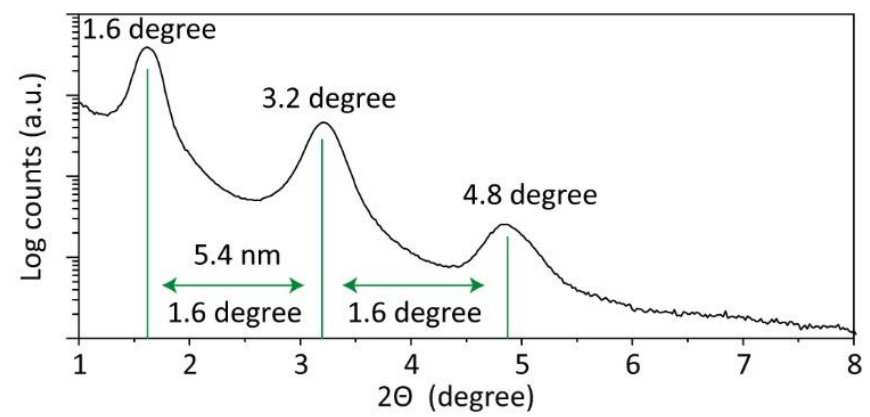

Figure S12. Low angle XRD of PbS NSs, indicating an average stacking thickness of $5.4 \mathrm{~nm}$. 
Supplementary high-resolution TEM and energy-dispersive X-ray spectroscopy analysis of PbS NSs. We carried out the analysis of the crystal structure (Figure $\mathrm{S}_{13}$ ) and composition (Figure $\mathrm{S}_{14}$ ) of the PbS NSs by high-resolution TEM (HRTEM) and energy-dispersive X-Ray spectroscopy (EDS).

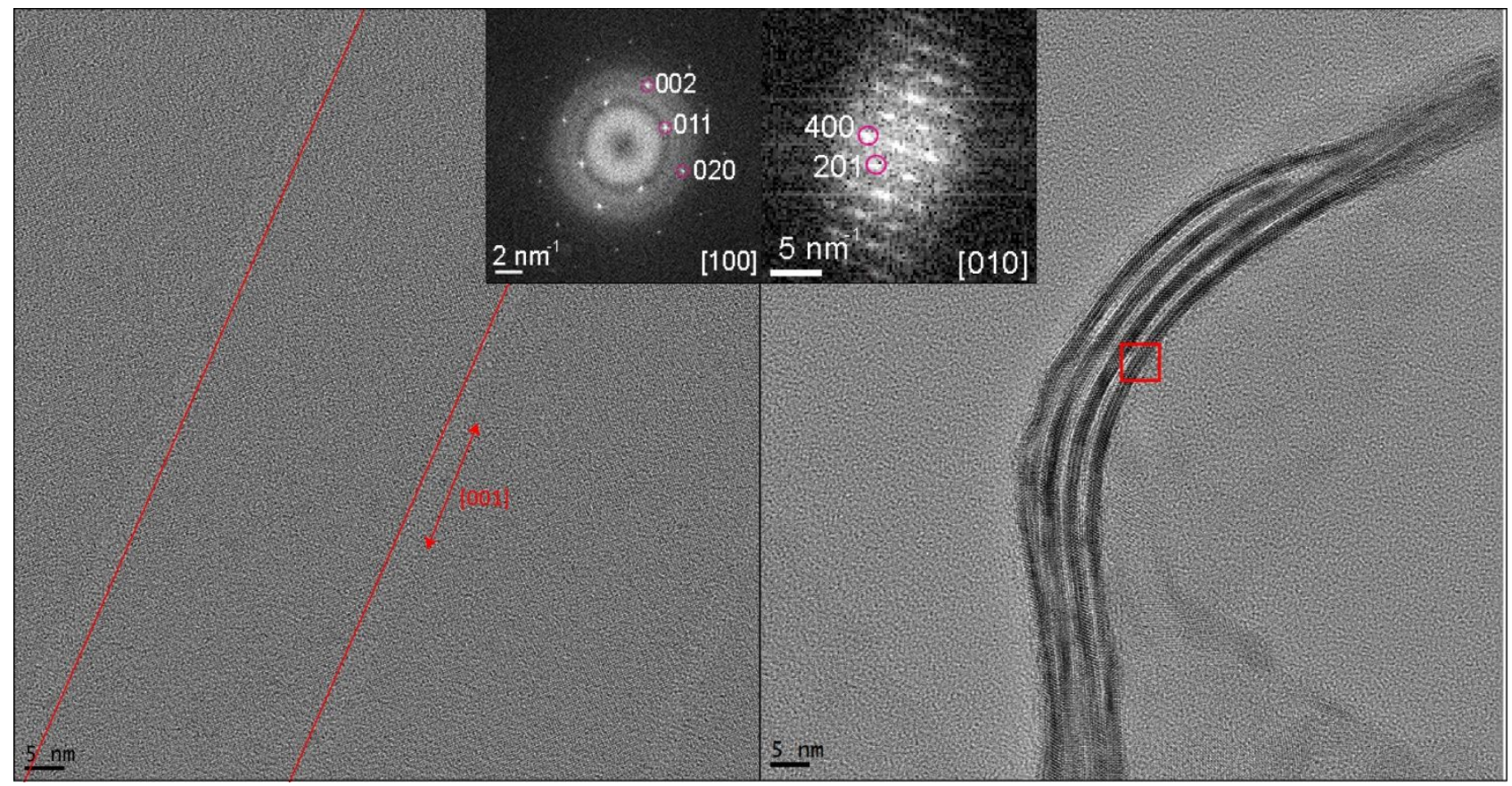

Figure S13. Additional HRTEM data. Left: Several ultrathin PbS NSs in their respective [10o] zone axes (sitting flat on the substrate). One is outlined by red lines and its corresponding FFT given in inset indicates that the NSs are elongates (grow preferentially) along their [oo1] direction, in agreement with the XRD data. Right: Four PbS NSs oriented side-on towards the viewing direction. An FFT generated from one of the NSs given in inset could only be indexed according to orthorhombic PbS structure.
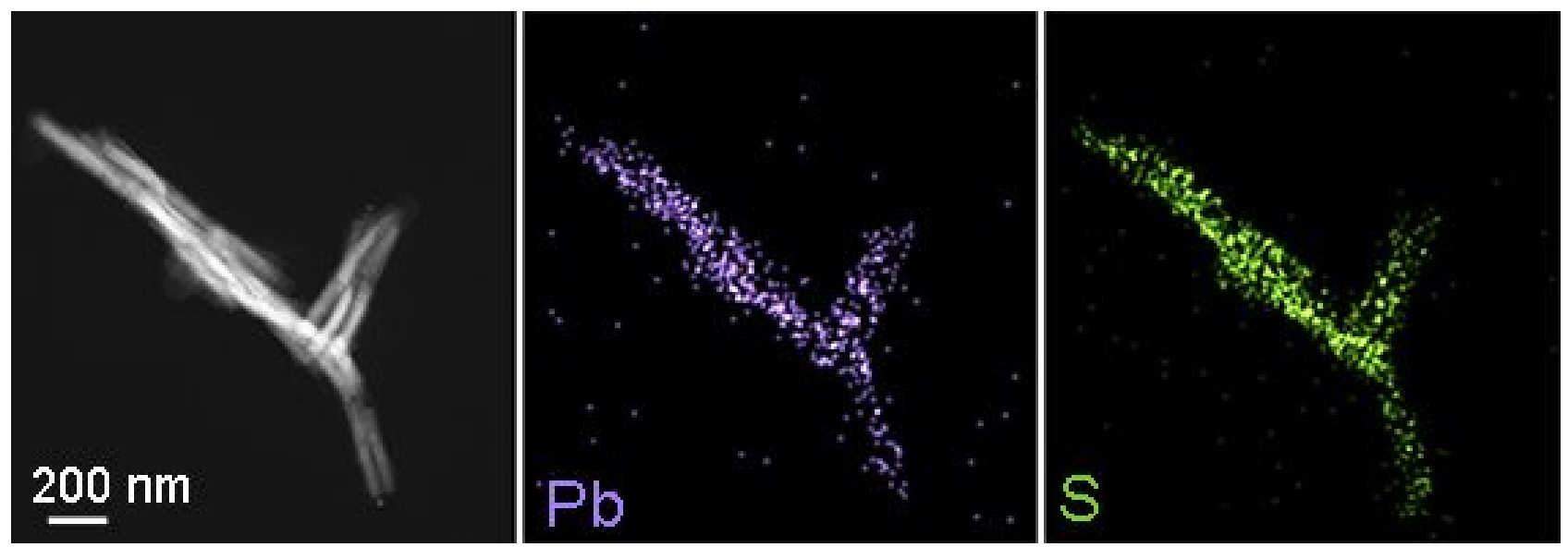

Figure S14. EDS elemental maps of PbS NSs. Left to right: Scanning TEM image of an aggregate of PbS NSs with the corresponding $\mathrm{Pb}$ and $\mathrm{S}$ elemental maps indicating uniform distribution of these elements within the NSs. 
Ligands effect on the NSs synthesis. To clarify the role of oleic acid (OA) and oleylamine (OLAM) in the NSs growth, we have carried out additional experiments by progressively increasing the amount of OA and OLAM, as reported in Table S2. First, it is important to note that we need both ligands, OLAM $(0.125 \mathrm{~mL})$ and $\mathrm{OA}(0.125 \mathrm{~mL})$, to dissolve the $\mathrm{Pb}(\mathrm{SCN})_{2}$. When lowering the concentration of either ligand (below $0.125 \mathrm{~mL}$ ), the $\mathrm{Pb}(\mathrm{SCN})_{2}$ does not completely dissolve (like for example $\mathrm{PbBr}_{2}$ ). Results shown in Figure $\mathrm{S}_{15}$, resulting from the variation of the amount of OA or OLAM, indicate that a slight (x2) or moderate $\left(\mathrm{x}_{5}\right)$ increase of OA or OLAM does not affect the synthesis much. The resulting PbS NSs keep theis sheet morphology and the orthorhombic crystal structure, but lateral sizes up to 6oox190 $\mathrm{nm}$ can be reached. However, going further by adding (x10) concentration of OA or OLAM, changes are clearer. A large excess of OA leads to the formation of polydisperse PbS NSs of uncontrollable size, with $\mathrm{PbS}$ rock-salt crystal structure. On the other hand, a large excess of OLAM promotes the appearance of nanocubes and PbS NSs with arrow-shape corners, hence the disappearance of the well-defined straight edges and sharp $90^{\circ}$ geometry, and also their crystal structure is the common PbS rock-salt. The fact that we obtain PbS NSs independently on the amount of $\mathrm{OA}$ and of $\mathrm{Pb}(\mathrm{SCN})_{2}$ we use, supports the key role of both species in the NSs growth, while the OLAM is probably just needed initially to dissolve the $\mathrm{Pb}(\mathrm{SCN})_{2}$. Indeed, amines bind strongly to $\mathrm{Pb}^{2+}$, therefore an excess of OLAM breaks apart the template and results in the formation of cubic PbS particles and distorted NSs. The addition of an excess of OA probably leads to a full replacement of $\mathrm{SCN}^{-}$in the template, again resulting in an unstable $\mathrm{Pb}$-oleate template, which promotes the formation of cubic (rock-salt) PbS nanomaterials. Our explanation is that the Pboleate forms a lamellar/layered template, which is further stabilized by the pseudo-halide $\mathrm{SCN}^{-}$ions, probably in a similar way that halide ions do in nanoplatelets syntheses., ${ }^{1,2}$ During the heating to the final reaction temperature $\left(150-180^{\circ}\right)$, the $\mathrm{Pb}$ oleate-SCN system decomposes, giving rise to the formation of the PbS NSs, similarly to the case of the Cu-thiolate-halides system, which evolves into ultrathin $\mathrm{Cu}_{2-\mathrm{x}} \mathrm{S} \mathrm{NSs},{ }^{3}$

Table S2. Reaction conditions tested from the typical synthesis (first row): $0.1 \mathrm{mmol} \mathrm{Pb}(\mathrm{SCN})_{2}$ in $10 \mathrm{~mL} \mathrm{ODE}$, 0.250 $\mathrm{mL} \mathrm{OA}$ and $0.125 \mathrm{~mL}$ OLAM at $165^{\circ} \mathrm{C}$ by modifying the OA and OLAM volume added in the reaction medium. The average dimensions indicated were estimated from the measurement of at least 30 NSs in each sample.

\begin{tabular}{|c|c|c|c|c|c|c|}
\hline $\begin{array}{c}\text { Pb(SCN) } \\
(\mathbf{m m o l})\end{array}$ & $\begin{array}{c}\text { Reaction } \\
\text { temperature }\left({ }^{\mathbf{o}} \mathbf{C}\right)\end{array}$ & $\begin{array}{c}\text { ODE } \\
(\mathbf{m L})\end{array}$ & $\begin{array}{c}\text { OLAM } \\
(\mathbf{m L})\end{array}$ & OA (mL) & quenching & Result \\
\hline 0.1 & 165 & 10 & 0.125 & 0.250 & Water bath & NSs with $180 \times 35 \mathrm{~nm}$ \\
\hline 0.1 & 165 & 5 & 0.250 & 0.250 & Water bath & NSs with $420 \times 75 \mathrm{~nm}$ \\
\hline 0.1 & 165 & 5 & 0.125 & 0.500 & Water bath & NSs with $370 \times 94 \mathrm{~nm}$ \\
\hline 0.1 & 165 & 10 & 0.625 & 0.250 & Water bath & NSs with $400 x 64 \mathrm{~nm}$ \\
\hline 0.1 & 165 & 10 & 0.125 & 1.250 & Water bath & NSs with 600x190 nm \\
\hline 0.1 & 165 & 5 & 1.25 & 0.250 & Water bath & $\begin{array}{c}\text { mix of nanocubes and } \\
\text { arrow-corner NSs }\end{array}$ \\
\hline 0.1 & 165 & 5 & 0.125 & 2.50 & Water bath & polydisperse NSs \\
\hline
\end{tabular}




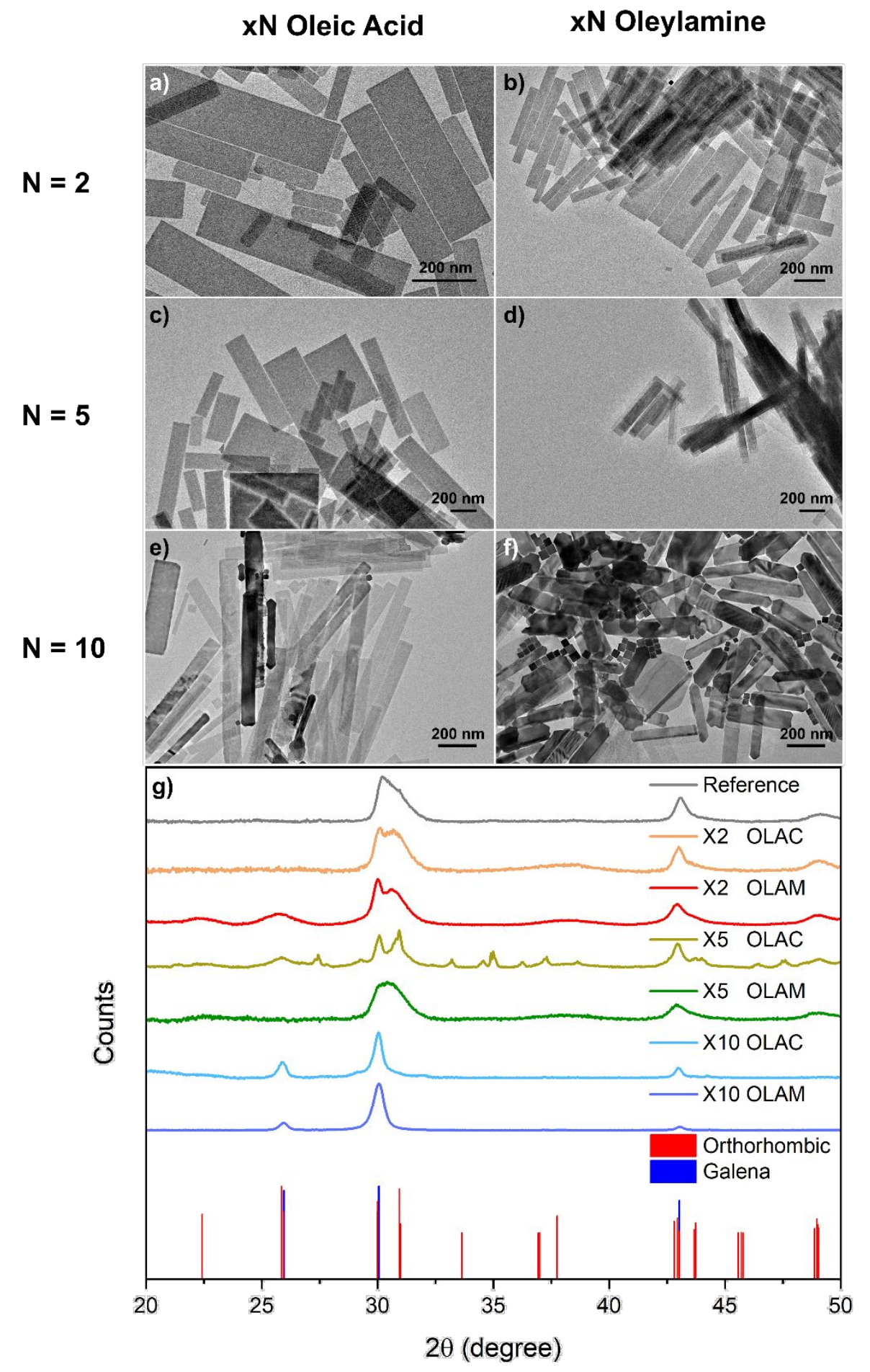

Figure S15. (a-f) Representative TEM images of the evolution of the PbS nanomaterials obtained by systematically modifying the OA and OLAM amount in the reaction medium, together with the corresponding XRD patterns (g). In (g) both PbS orthorhombic (calculated pattern - see CIF file) and rock-salt bulk diffractions are also reported (ICSD card 53932). 
Fourier-transform infrared (FTIR) spectroscopy on PbS NSs. In order to gain insight into the presence and type of ligands that passivate the surface of the PbS NSs after synthesis, we carried out FTIR spectroscopy characterization. As is shown in Figure S16, the spectrum of PbS NSs exhibits a broad signal at $2040 \mathrm{~cm}^{-1}$ which can be attributed to surface bound thiocyanate groups. ${ }^{4}$ In addition, carboxylate signatures at 1520 and $1410 \mathrm{~cm}^{-1}$ are also visible, indicating the presence of oleate moieties. 5 Finally, the absence of the carbonyl $(C=O)$ signal characteristic of oleic acid and the absence of oleylamine signatures at 790 and $970 \mathrm{~cm}^{-1}$ suggest that these ligands are absent.
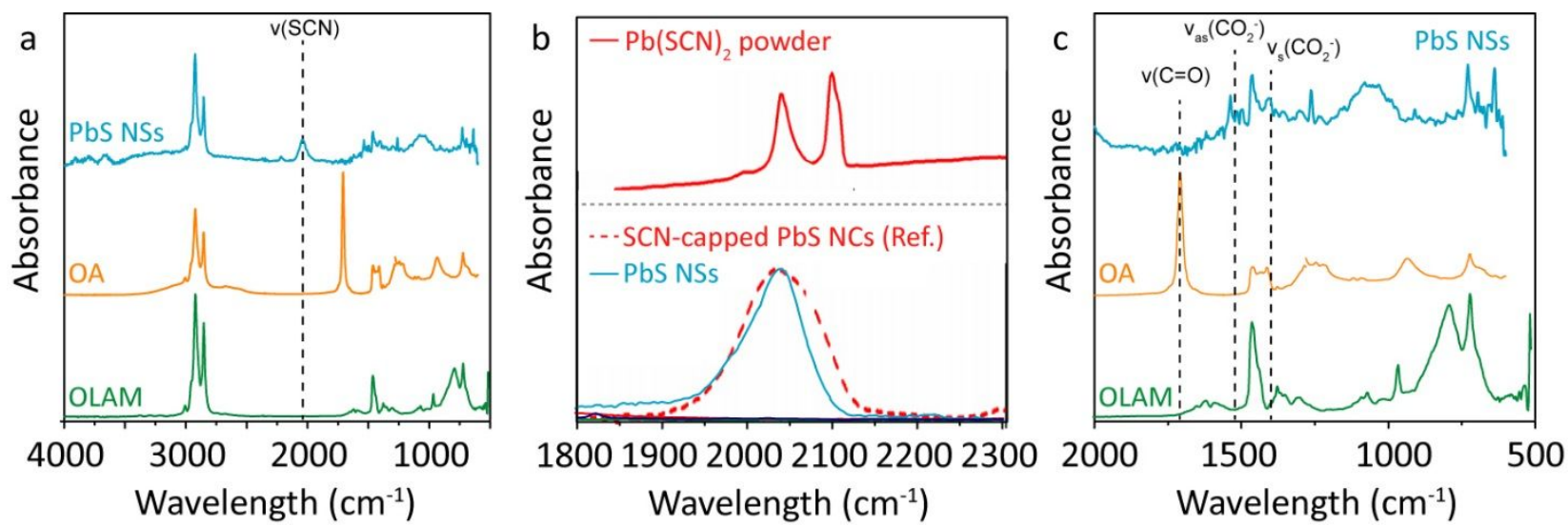

Figure S16. FTIR spectra from PbS NSs. (a) FTIR spectra of PbS NSs, oleylamine (OLAM) and oleic acid (OA). (b) FTIR spectrum of lead thiocyanate $\left(\mathrm{Pb}(\mathrm{SCN})_{2}\right)$ compared to that of $\mathrm{PbS} \mathrm{NSs}$ and that of thiocyanate-capped $\mathrm{PbS}$ nanocrystals ${ }^{4}$. (c) Narrowed FTIR spectra of PbS NSs, oleylamine (OLAM) and oleic acid (OA) highlighting the presence of carboxylate moieties $\left(\mathrm{CO}_{2}^{-}\right)^{5}$ and the absence of carbonyl $(\mathrm{C}=\mathrm{O})$ resonances in the spectrum of $\mathrm{PbS}$ NSs.

Complementary optical and band structure data by DFT calculations

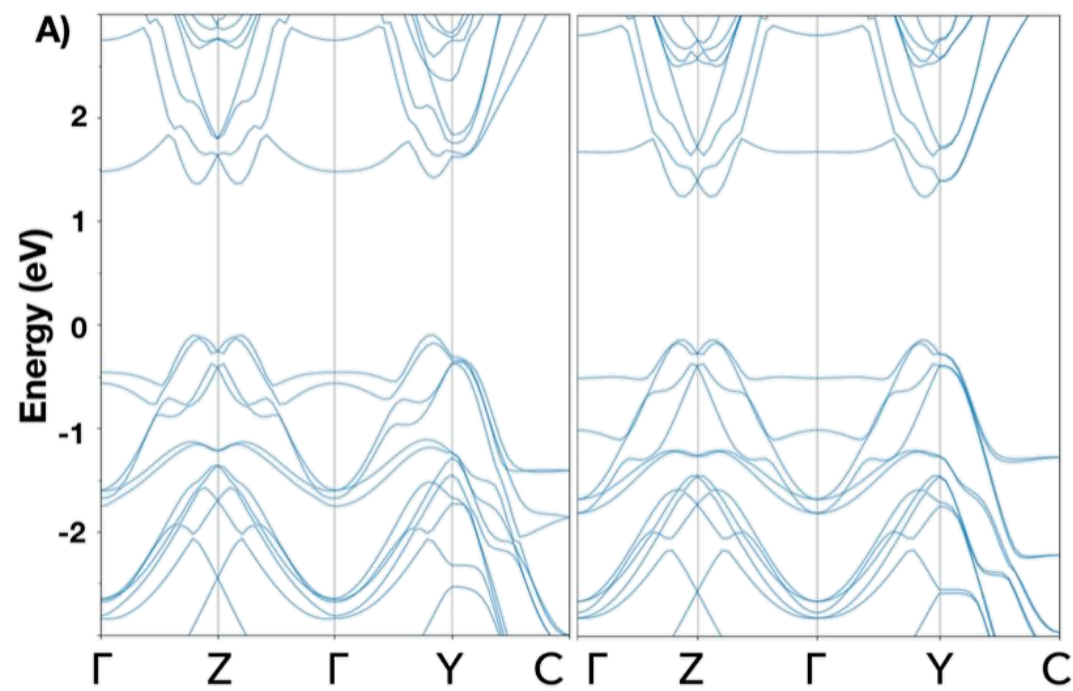

B)

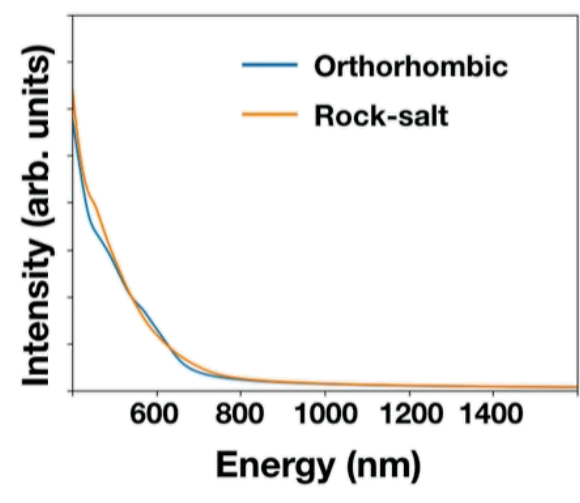

Figure S17. Optical data and DFT calculations. a) Band structure of orthorhombic (left side) and rock-salt (right) side computed at the DFT/PBE theory. b) The corresponding absorption spectrum computed at the DFT/PBE level of theory. 
Post-synthesis surface treatment towards PbS NSs photoluminescence properties. In an attempt to activate the possible photoluminescence (PL) from the obtained PbS NSs by improving the surface passivation, we carried out a chloridebased post-synthetic treatment, as reported for other nanomaterials. ${ }^{6}$ Therefore, we prepared a mixture of $\mathrm{PbCl}_{2} / \mathrm{OLAM} /$ tetradecylphosphonic acid-TDPA- $(0.33 \mathrm{M})$ at $120^{\circ} \mathrm{C}$ instead of the $100^{\circ} \mathrm{C}$ reported temperature, to ensure the dissolution of the $\mathrm{PbCl}_{2}$ in OLAM/TDPA medium and avoid the $\mathrm{PbCl}_{2}$ precipitation after cooling down the solution to $60^{\circ} \mathrm{C}$ for performing the treatment.7 Two sequences of experiments have been performed, by modifying the time (from 1 to $60 \mathrm{~min}$ ) and the amount of ligands added (from 100 to $500 \mu \mathrm{L}$ of $0.33 \mathrm{M} \mathrm{PbCl}_{2} / \mathrm{TDPA} / \mathrm{OLAM}$ for each $150 \mu \mathrm{L}$ of a PbS NSs dispersion in toluene $\left(16 \mathrm{mg} \mathrm{mL}^{-1}\right)$ in $1 \mathrm{~mL}$ of toluene). As illustrated in Figure S18a-f, the TEM images show the formation of dots decorating the $\mathrm{PbS}$ NSs in all the cases, an indication of high surface reactivity, together with a slight change in the sheets morphology, with the disappearance of the sharp edge/corners towards a more round shape. When evaluating the PL properties in solution, we observed a PL emission in the range of $1110-1180 \mathrm{~nm}$, which shifted with the time or the amount of $\mathrm{PbCl}_{2} / \mathrm{OLAM} / \mathrm{TDPA}$ added during the surface treatment (Figure S18g-h). Considering that (i) the absorbance spectrum of the starting PbS NSs is up to $800 \mathrm{~nm}$, (ii) the theoretically calculated excitonic peak is at $620 \mathrm{~nm}$, and (iii) the size of the dots formed at their surface as result of the treatment is ca. 3-4 nm diameter (determined by TEM measurements), we can ascribe the observed PL emission to the $\mathrm{PbS}$ dots forming during the treatment, as a consequence of etching of the NSs. This is further confirmed by noticing that a Stokes shift of ca. $530 \mathrm{~nm}$ for the PbS NSs is completely unexpected even comparing with the literature (e.g. ca. $18 \mathrm{~nm}$, see Khan et al. ${ }^{8}$; ca. $200 \mathrm{~nm}$, see Bhandari et al. ${ }^{9}$ or ca. $150 \mathrm{~nm}$, see Zhang et al. ${ }^{10}$ Chem. Mater. 2016, 28, 127), while a PL emission of PbS QDs of 3-4 nm diameter at ca. $1150 \mathrm{~nm}$ matches well with the literature ${ }^{7}$ and also with our results from PbS QDs synthesis using different precursors, as shown in Figure S18i.
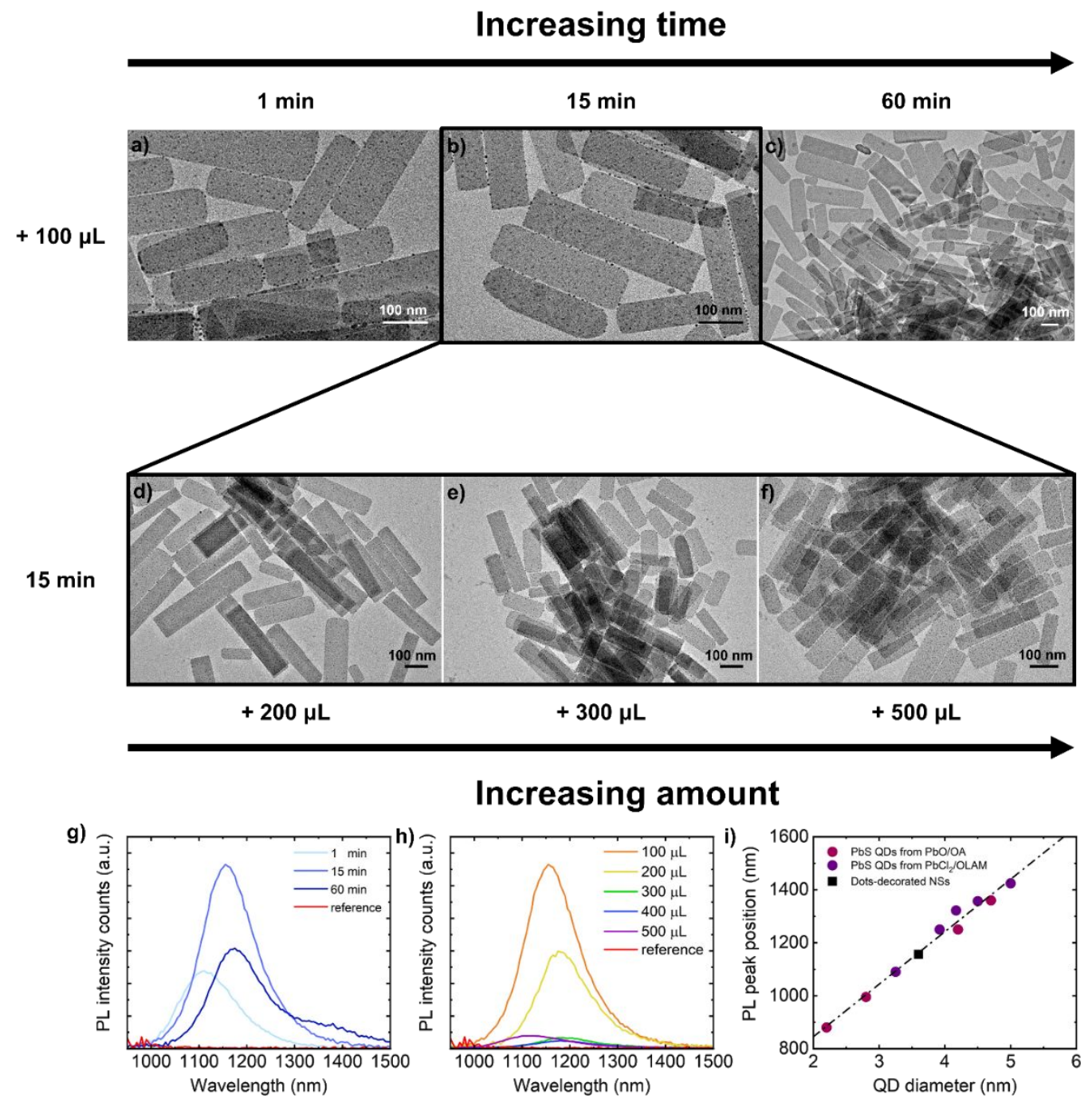

Figure S18. PbS NSs surface treatments. Representative TEM images of the PbS NSs obtained after the $\mathrm{PbCl}_{2} / \mathrm{TDPA}_{\mathrm{OLAM}}$ surface treatment, by modifying the reaction time (100 $\mu \mathrm{L}$ of ligands) (a-c) and the ligands added (fixing $15 \mathrm{~min}$ ) (d-f). Evolution of the corresponding PL emission spectra of the PbS NSs obtained after the same surface treatment by modifying the time (g) and the ligands added (h). The PbS NSs were dispersed in TCE and $400 \mathrm{~nm}$ was used as excitation wavelength 
(Xe lamp). (i) Photoluminescence emission peak position for different size PbS QDs synthesized in our lab from $\mathrm{PbO} / \mathrm{OA}^{11}$ and $\mathrm{PbCl}_{2} / \mathrm{OLAM}^{7,12}$ following the protocols established in the literature indicated.

In a last attempt to avoid the formation of dots, alternative but similar surface treatments have been performed. In practice, $\mathrm{PbCl}_{2} / \mathrm{OA} / \mathrm{TDPA}, \mathrm{PbO} / \mathrm{OA}$ and $\mathrm{PbO} / \mathrm{OA} / \mathrm{TDPA}$ fixing the reaction conditions: $100 \mu \mathrm{L}$ of solution ligands added, $60^{\circ} \mathrm{C}$ and 15 min. Results indicate that we successfully minimized the formation of dots in the first and third cases (Figure Sig). Unfortunately no PL emission from the resulting PbS NSs has been observed in any case.
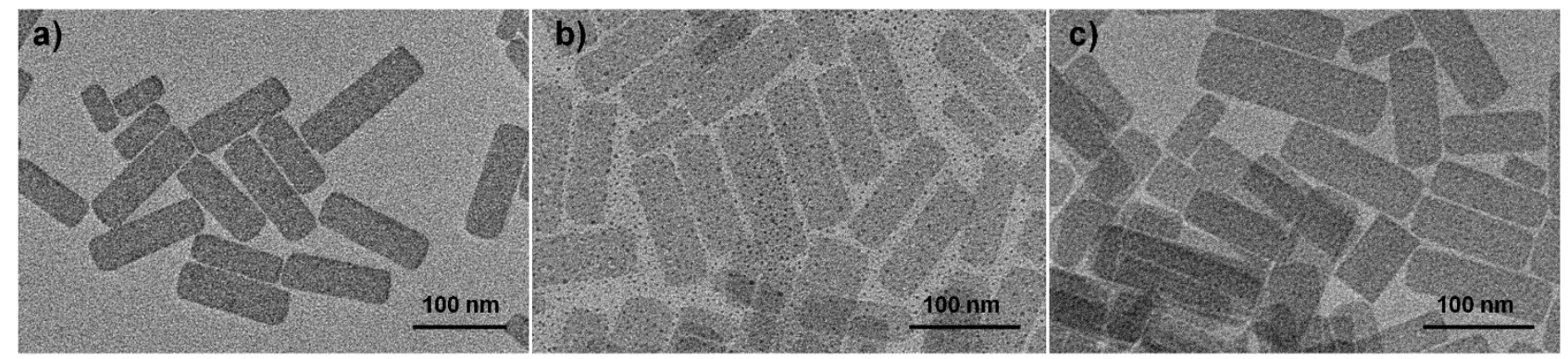

Figure S19. PbS NSs alternative surface treatments. Representative TEM images of the PbS NSs obtained after the surface treatments with $\mathrm{PbCl}_{2} / \mathrm{TDPA} / \mathrm{OA}, \mathrm{PbO} / \mathrm{OA}$ and $\mathrm{PbO} / \mathrm{TDPA} / \mathrm{OA}$.

\section{Complementary characterization of films and devices.}

As is shown in Figure S2o, the PbS NS films preserve their structural and optical properties after the ligand exchange and annealing steps (films deposited on glass and treated using the same protocol as described in the main text: ligand exchange performed with a methanol solution containing 1-ethyl-3-methylimidazolium iodide and the film was annealed at $8 \mathrm{o}^{\circ} \mathrm{C}$ under inert atmosphere).
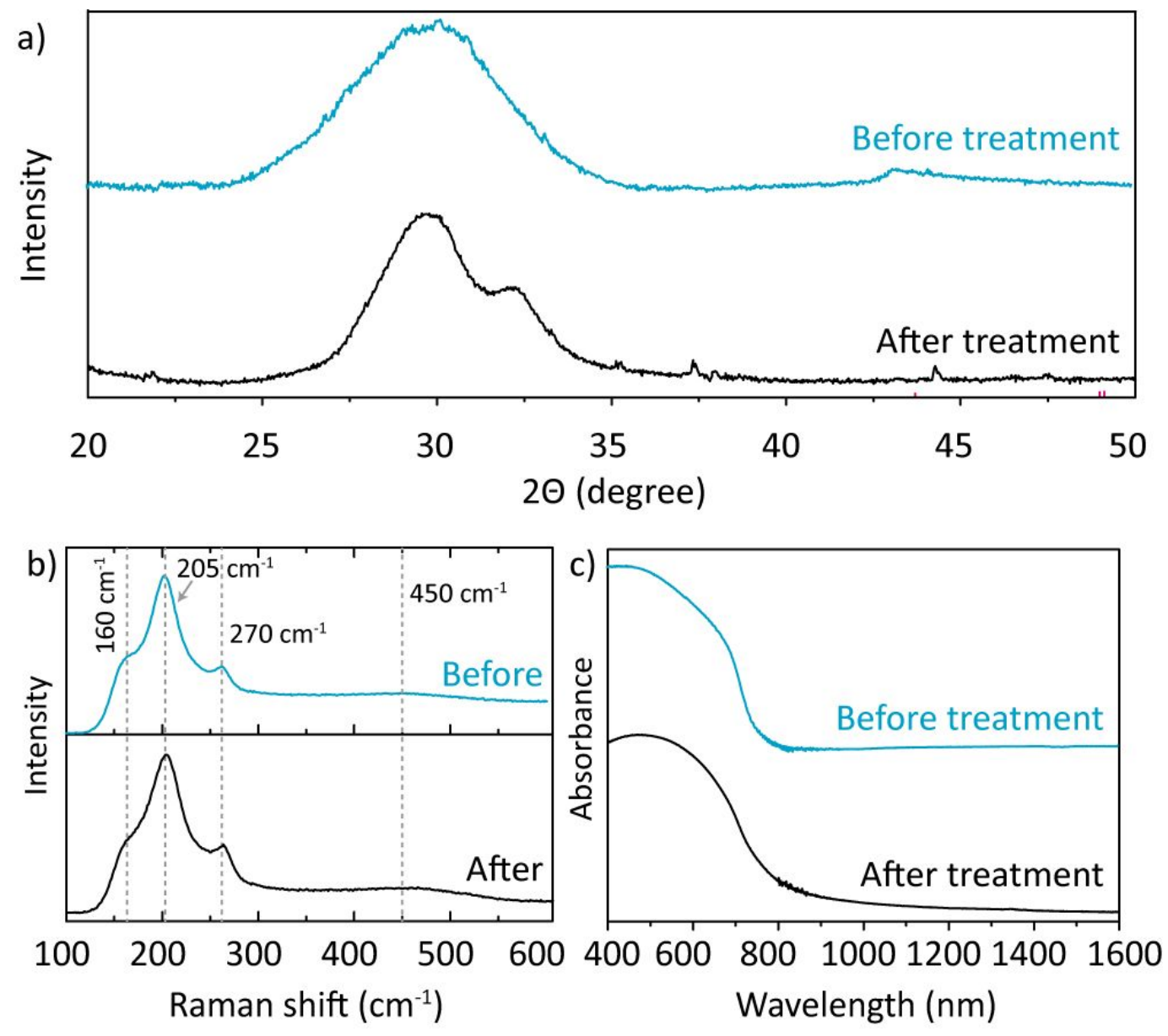
Figure S2o. Structural and optical properties of PbS NSs films before and after treatment. a) XRD patterns, b) Raman spectra and c) absorbance spectra of PbS NSs films before and after treatment. The film treatment consists of solid-ligand exchange with 1-ethyl-3-methylimidazolium iodide (in methanol) followed by annealing at $80{ }^{\circ} \mathrm{C}$ (inert atmosphere). We note that the XRD peaks become slightly more defined probably as result of the film annealing and no significant changes in optical properties are observed.

We also prepared PbS NS-based photoconductor devices on silicon substrates ( $\mathrm{Si} / \mathrm{SiO}_{2}$ thermally growth $300 \mathrm{~nm}$, University wafer $^{\circ}$ ) using interdigitated electrodes (Ti/Au) with $10 \mu \mathrm{m}$ gap, see Figure S21a. From their ON/OFF response (Figure S16b) under white LED light (400-750 $\mathrm{nm}$ range and a power density of $100 \mathrm{~mW} \mathrm{~cm}^{-2}$ ) we estimated a responsivity (R) of $0.1 \mathrm{~A} \mathrm{~W}^{-1}$ and a detectivity (D) of 1.3.10 ${ }^{9}$ Jones.
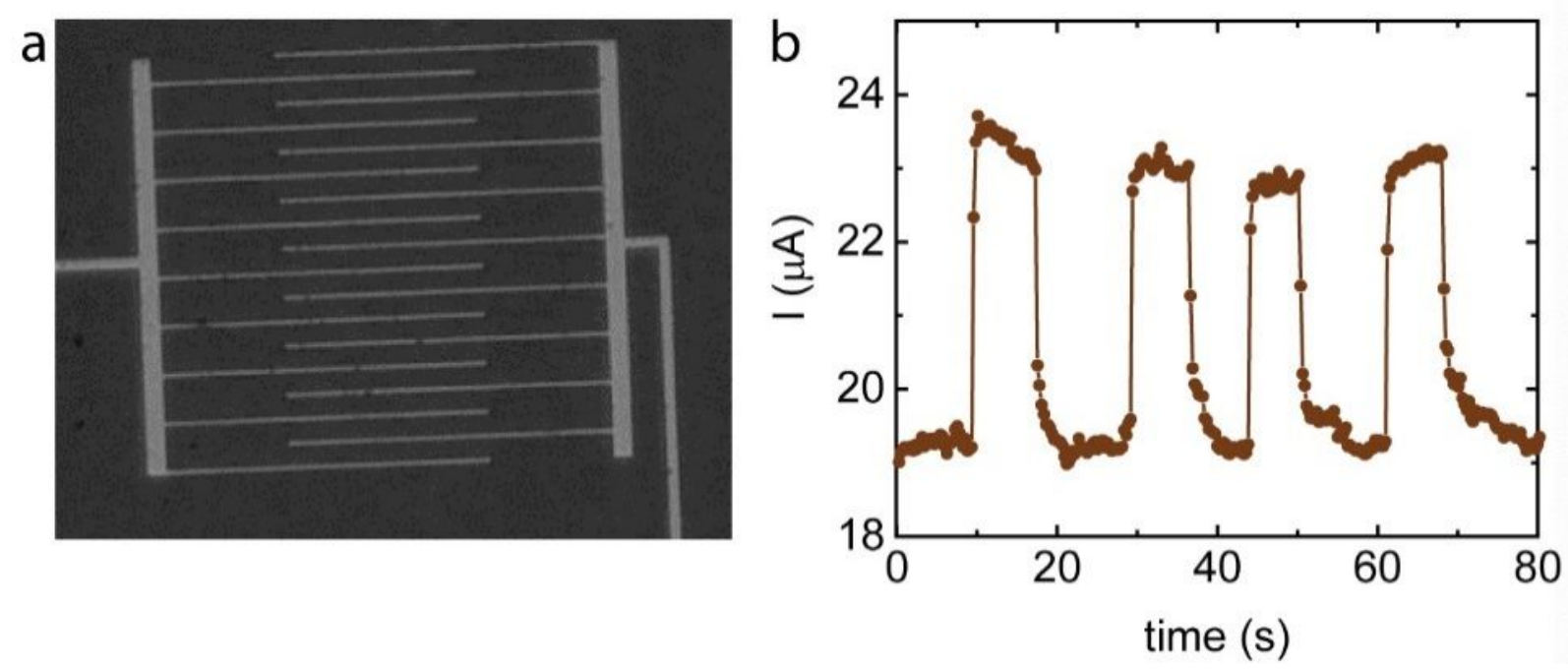

Figure S21. Additional devices characterization. a) Optical microscope image of the Ti/Au interdigitated contacts (10 $\mu \mathrm{m}$ gap) on silicon substrate. b) ON/OFF response of the PbS NS-based device under white LED light (400-750 nm range and a power density of $100 \mathrm{~mW} \mathrm{~cm} \mathrm{~cm}^{-2}$ ) built with 7 layers using iodine-ligand exchange on top of the interdigitated electrodes applying $20 \mathrm{~V}$.

The device performance over storage time is shown in Figure S22 in terms of normalized responsivity. The PbS NC-based devices are more stable than the PbS NS ones, nevertheless, we note that the performance of PbS NS device stabilizes after 4 weeks of storage. 


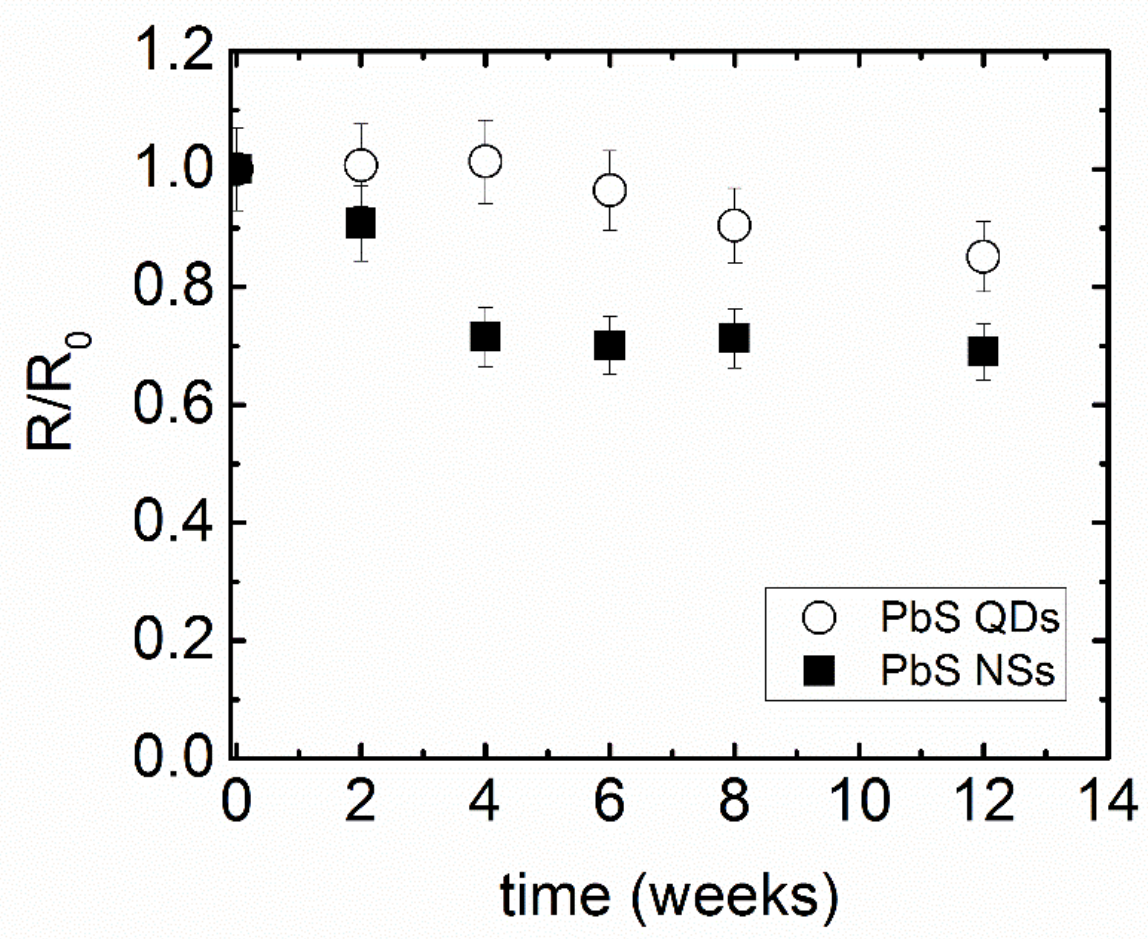

Figure S22 - Device performance over storage time. Normalized responsivity $\left(R / R_{0}, R_{o}\right.$ denotes the responsivity at day o) of devices fabricated with PbS NSs (squares) or PbS NCs (circles), on glass using shadow mask patterning (100 $\mu \mathrm{m}$ pads gap). The devices were stored in the dark under ambient conditions (relative humidity $<30 \%$ ). Values shown are the average results from the monitoring of 3 different devices per sample.

The SEM images in Figure S23 correspond to a PbS NCs film from a mask-shadow device on glass (100 $\mu \mathrm{m}$ pads gap). The formation of small cracks can be observed at higher magnification (Figure S23b).

a)

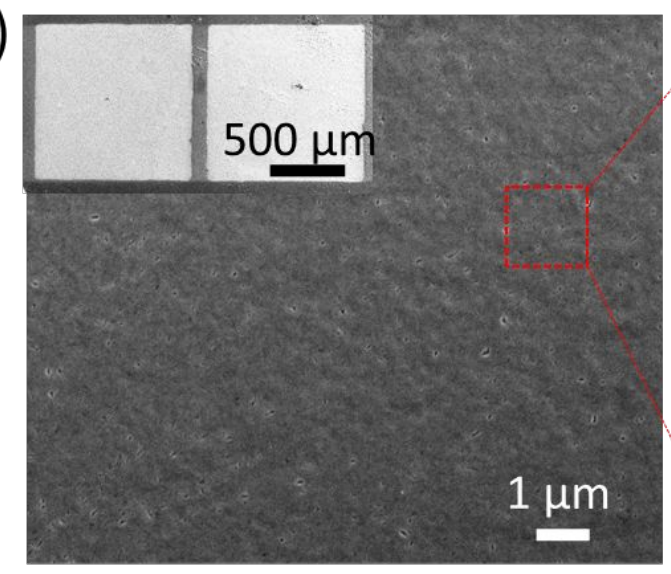

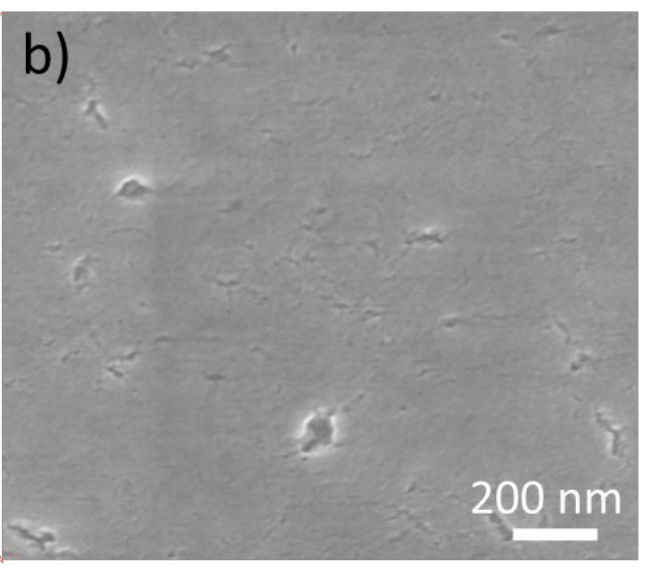

Figure S23. Representative SEM images of the PbS NC film from a mask-shadow device (inset) at different magnifications.

SEM images of PbS NC films on PET substrates after 5 bending cycles (Figure S24c-d) reveal the formation of deep cracks, while PbS NS film remain more connected (Figure S24a-b). 

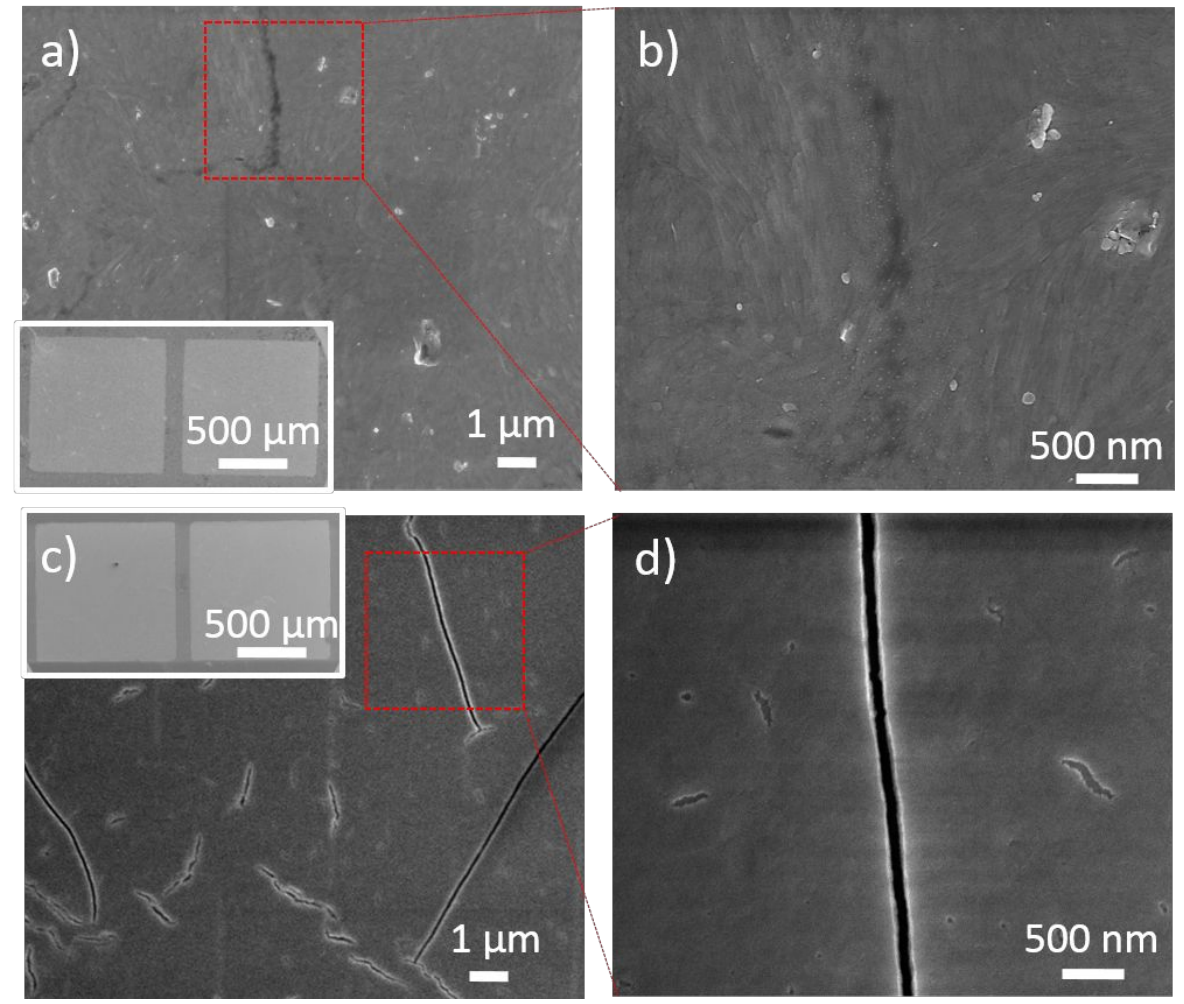

Figure S24. Representative SEM images at different magnifications of PbS NS film (a, b) and PbS NC film (c, d) from maskshadow devices (inset) made on PET substrates after 5 bending cycles. 


\section{$\underline{\text { References }}$}

(1) Gerdes, F.; Navío, C.; Juárez, B. H.; Klinke, C. Size, Shape, and Phase Control in Ultrathin CdSe Nanosheets. Nano Lett. 2017, 17 (7), 4165-4171. https://doi.org/10.1021/acs.nanolett.7boo937.

(2) Christodoulou, S.; Climente, J. I.; Planelles, J.; Brescia, R.; Prato, M.; Martín-García, B.; Khan, A. H.; Moreels, I. Chloride-Induced Thickness Control in CdSe Nanoplatelets. Nano Lett. 2018, 18 (10), 6248-6254.

https://doi.org/10.1021/acs.nanolett.8bo2361.

(3) van der Stam, W.; Akkerman, Q. A.; Ke, X.; van Huis, M. A.; Bals, S.; de Mello Donega, C. Solution-Processable Ultrathin Size- and Shape-Controlled Colloidal $\mathrm{Cu}_{2-x}$ S Nanosheets. Chem. Mater. 2015, 27 (1), $283^{-291 .}$

https://doi.org/10.1021/cm503929q.

(4) Koh, W.; Saudari, S. R.; Fafarman, A. T.; Kagan, C. R.; Murray, C. B. Thiocyanate-Capped PbS Nanocubes: Ambipolar Transport Enables Quantum Dot Based Circuits on a Flexible Substrate. Nano Lett. 2o11, 11 (11), 4764-4767. https://doi.org/10.1021/nl202578g.

(5) Tackett, J. E. FT-IR Characterization of Metal Acetates in Aqueous Solution. Appl. Spectrosc. 1989, 43 (3), $483-489$.

(6) Page, R. C.; Espinobarro-Velazquez, D.; Leontiadou, M. A.; Smith, C.; Lewis, E. A.; Haigh, S. J.; Li, C.; Radtke, H.; Pengpad, A.; Bondino, F.; et al. Near-Unity Quantum Yields from Chloride Treated CdTe Colloidal Quantum Dots. Small 2015, 11 (13), 1548-1554. https://doi.org/10.1002/smll.201402264.

(7) Moreels, I.; Justo, Y.; De Geyter, B.; Haustraete, K.; Martins, J. C.; Hens, Z. Size-Tunable, Bright, and Stable PbS Quantum Dots: A Surface Chemistry Study. ACS Nano 2011, 5 (3), 2004-2012. https://doi.org/10.1021/nn103050w.

(8) Khan, A. H.; Brescia, R.; Polovitsyn, A.; Angeloni, I.; Martín-García, B.; Moreels, I. Near-Infrared Emitting Colloidal PbS Nanoplatelets: Lateral Size Control and Optical Spectroscopy. Chem. Mater. 2017, 29 (7), 2883-2889. https://doi.org/10.1021/acs.chemmater.6bo5111.

(9) Bhandari, G. B.; Subedi, K.; He, Y.; Jiang, Z.; Leopold, M.; Reilly, N.; Lu, H. P.; Zayak, A. T.; Sun, L. ThicknessControlled Synthesis of Colloidal PbS Nanosheets and Their Thickness-Dependent Energy Gaps. Chem. Mater. 2014, 26 (19), 5433-5436. https://doi.org/10.1021/cm502524z.

(10) Zhang, H.; Savitzky, B. H.; Yang, J.; Newman, J. T.; Perez, K. A.; Hyun, B.-R.; Kourkoutis, L. F.; Hanrath, T.; Wise, F. W. Colloidal Synthesis of PbS and PbS/CdS Nanosheets Using Acetate-Free Precursors. Chem. Mater. 2016, 28 (1), 127-134. https://doi.org/10.1021/acs.chemmater.5bo3348.

(11) Barkhouse, D. A. R.; Debnath, R.; Kramer, I. J.; Zhitomirsky, D.; Pattantyus-Abraham, A. G.; Levina, L.; Etgar, L.; Grätzel, M.; Sargent, E. H. Depleted Bulk Heterojunction Colloidal Quantum Dot Photovoltaics. Adv. Mater. 2011, 23 (28), 3134-3138. https://doi.org/10.1002/adma.201101065.

(12) Moreels, I.; Lambert, K.; Smeets, D.; De Muynck, D.; Nollet, T.; Martins, J. C.; Vanhaecke, F.; Vantomme, A.; Delerue, C.; Allan, G.; et al. Size-Dependent Optical Properties of Colloidal PbS Quantum Dots. ACS Nano 2009, 3 (10), 30233030. https://doi.org/10.1021/nngoo863a. 